01

\title{
Численное решение задачи определения температурной зависимости теплофизических параметров твердых сред
}

\author{
(C) А.А. Обухов, ${ }^{1,2}$ Т.А. Новикова, ${ }^{1,3}$ В.Г. Лебедев, ${ }^{1,2,3}$ В.И. Ладьянов, ${ }^{2}$ А.В. Обухов ${ }^{1}$ \\ ${ }^{1}$ АО НПО МКМ, \\ 426058 Ижевск, Россия \\ ${ }^{2}$ Научный центр МФМ УдмФИЦ УрО РАН, \\ 426067 Ижевск, Россия \\ ${ }^{3}$ Удмуртский государственный университет, \\ 426034 Ижевск, Россия \\ e-mail: obuh87@bk.ru
}

Поступило в Редакцию 12 февраля 2020 г.

В окончательной редакции 30 апреля 2020 г.

Принято к публикации 3 мая 2020 г.

Рассмотрены два различных подхода для определения теплофизических свойств исследуемого материала. Необходимые экспериментальные данные по термическим кривым были получены из имитационного моделирования процесса распространения тепла на основе численного решения одномерной задачи уравнения теплопроводности. Значения коэффициентов теплопроводности и температуропроводности рассчитаны независимо друг от друга по предлагаемым методикам. Результат исследования позволяет оценить погрешность полученных параметров за счет сравнения с данными, заложенными в имитационное моделирование. Предлагаемая методика дает качественное и количественное представления о теплофизических свойствах материалов.

Ключевые слова: численный эксперимент, температура, уравнение теплопроводности, теплофизические свойства.

DOI: $10.21883 /$ JTF.2020.12.50115.50-20

\section{Введение}

Развитие современных технологий тесно связано с новыми материалами. Довольно часто их использование в различных областях, определяется уникальными теплофизическими характеристиками. Методики оценки коэффициента теплопроводности и других теплофизических свойств материалов широко обсуждаются в литературе. Заявленные значения теплофизических параметров иногда вызывают сомнения, поскольку могут сильно отличаться по данным разных источников, или полностью отсутствовать. Также авторы не всегда сообщают о характеристиках предлагаемого материала, например, дисперсности, структуре, их температурных зависимостях и т.д. В результате расширения спектра материалов появляются новые методы измерения теплофизических параметров, которые отличаются временем и точностью измерения, разновидностями исследуемых образцов и диапазонами температур [1-7].

В области литейных технологий существуют ситуации, требующие быстрого и эффективного определения теплофизических параметров среды. Например, литейная форма, в которую попадает расплавленный металл, как правило, является расходным материалом. С одной стороны, существенным плюсом является низкая цена производства таких форм, снижающая конечную стоимость изделия. С другой стороны - теплофизические характеристики материалов могут довольно существенно меняться, что сильно влияет на качество конечного продукта, особенно при точном литье. Как правило, материал формы является мелкодисперсной смесью твердых непроводящих частиц с различными свойствами, поэтому теплофизические характеристики формы достаточно сложно прогнозировать. Естественно, что такие материалы не являются прерогативой только литейных технологий [1].

Целью настоящей работы является теоретическая разработка методики определения теплофизических параметров твердой непроводящей среды. Необходимые данные для отработки методики и дальнейшей постановки натурного эксперимента были взяты из математического моделирования, основанного на численном решении уравнения теплопроводности для одномерного случая.

\section{1. Методы измерений и состояние проблемы}

На сегодняшний день существуют два основных метода измерения коэффициента теплопроводности, в которых используются стационарный и нестационарный (или переходный) режимы [8]. Далее для упрощения будем называть измерения, выполненные при стационарном и нестационарном (переходном) режимах, стационарным и переходным методами соответственно. Каждый из этих методов основан на фундаментальных законах тепло- 
проводности и электрических аналогиях и подходит для ограниченного набора материалов. Существуют важные различия между стационарными и переходными методами $[9,10]$.

Для измерения в твердых материалах стационарные методы считаются более точными, чем переходные, но в то же время стоить отметить, что переходные методы позволяют определять коэффициент температуропроводности [11].

В стационарном методе измерение производится в тот момент, когда тепловое состояние исследуемого материала достигает полного равновесия [10]. Необходимо продолжительное время проведения эксперимента для достижения данного состояния $[9,10]$. Под равновесием понимается состояние, когда температура в каждой точке образца постоянна и не изменяется со временем. Использование такого метода ограничено дорогостоящим, хорошо спроектированным экспериментальным оборудованием, которое позволяет отслеживать и контролировать равновесные состояния. Тем не менее такой подход наиболее распространен и считается наиболее точным методом измерения.

Коэффициенты теплопроводности в переходном методе определяются во время процесса нагрева или остывания с помощью специальных датчиков. Эти измерения могут быть сделаны относительно быстро, что дает значительное преимущество перед другими измерениями, основанными на стационарных методах $[9,10,12]$. С этой целью используются многочисленные решения нестационарного уравнения теплопроводности для одно-, двух-, трехмерных геометрий измерительных приборов [12]. В качестве измерительных датчиков в переходных методах обычно используются игольчатые зонды (термопары) [9]. Естественно, что характеристики таких термопар являются значимыми и влияющими на точность измерений. Неоспоримым преимуществом измерений, выполненных в переходном методе, является существенное по сравнению со стационарными методами уменьшение времени проведения эксперимента. Сравнение наиболее распространенных методов измерения теплопроводности можно найти в работе [12].

Одно из основных препятствий, мешающих быстрому и надежному измерению теплофизических свойств, связано с необходимостью решения обратной задачи теплопроводности: определению теплофизических параметров по распределению температуры. Эта задача восходит еще к работам Самарского А.А. и Вабищевича П.Н. [2], Зарубина В.С. [3], Алифанова О.М. [4] и многих других. Из более современных подходов следует отметить работы Формалева В.Ф. [5-7], нацеленные на определении теплофизических параметров анизотропных сред с помощью минимизации функционала невязки на основе программных средств. Последнее требует от экспериментатора как определенных ресурсов для их реализации, так и достаточно высоких навыков работы с прикладными программами.
По сути, целевой функцией научных исследований [2-7] является достижение максимальной точности измеряемых теплофизических параметров материалов. При этом нашу работу можно рассматривать как прагматическое дополнение к фундаментальным достижениям [2-7]. Она ориентирована на смену парадигмы измерений, когда во главу угла ставится быстрое и эффективное определение теплофизических параметров материалов, точность измерений которых должна быть достаточной для проведения технологических расчетов в рамках соответствующих вычислительных программ [13], но в то же время это не является самоцелью. Поэтому можно говорить о том, что предлагаемая концепция является не только научной, но и инженерной - требующей компромисса в достижении точности измерений, эффективности и возможности ее реализации в локальной области производства. Кроме того, использование имитационного моделирования дает возможность разделить возможные ошибки при проведении натурного эксперимента и ошибки, связанные с теоретической методикой.

В настоящей работе предлагается метод измерения, который дает достаточно достоверные оценки, позволяющие на их основе моделировать технологические процессы литья в известных на сегодня программных продуктах [13], а не уникальные по точности результаты. На практике точность математических моделей, используемых в программных продуктах, не превышает величину порядка 5-10\%. Поэтому вряд ли имеет смысл проводить теплофизические измерения для технологических процессов с более высокой точностью. Гораздо более существенным обстоятельством является возможность измерить на месте теплофизические параметры материалов, используемых на производстве. В идеале при наличии реального прибора можно было бы говорить об экспресс-анализе теплофизических свойств.

Другое ограничение, возникающее в рамках изложенной концепции - количество датчиков (термопар). Их установка приводит к дополнительному усложнению эксперимента, внесению новых погрешностей, затягиванию по времени. Минимально возможное число термопар, из которых удается извлечь достаточную информацию, оказалось равно четырем, поэтому далее мы рассматриваем именно этот случай.

Последний момент связан с тем, что проводится имитационное моделирование, а не реальный эксперимент. Чтобы имитировать процесс распространения тепла в некоторой сложной среде, мы используем известные a priori зависимости теплофизических параметров среды от температуры. На их основе проводится моделирование процесса распространения тепла от источника. Зная температурные зависимости в четырех заранее выбранных точках расположения „виртуальных“ термопар, можно количественно оценить зависимость теплофизических параметров сплошной среды от температуры.

Далее в настоящей работе представлены независимые друг от друга методики вычисления теплофизических 
параметров (коэффициентов температуропроводности и теплопроводности). Значения теплофизических коэффициентов для численного моделирования распространения тепла в непроводящей твердой среде с нелинейной зависимостью теплофизических коэффициентов от температуры были взяты из базы данных материалов, принадлежащих $\mathrm{AO}$ „НПО МКМ“, являющегося разработчиком пакета LVMFlowCV [13].

\section{2. Имитация нагрева материала в численном эксперименте}

Для получения необходимых исходных данных в виде термических кривых были проведены численные эксперименты с нагревом исследуемого материала. На данном этапе материал характеризуется известными теплофизическими параметрами, используемыми для расчетов. Физико-математическая модель нагрева основана на уравнении теплопроводности в следующей постановке.

Рассмотрим одномерный стержень длинной $L$, с начальной температурой $T(x, 0)=T_{0}$, нагревателем с мощностью $P$ в точке $x=0$ и условием постоянства температуры на границе области $T(L, t)=T_{0}$. Распределение температур в стержне с течением времени определяется решением следующей краевой задачи для уравнения теплопроводности с коэффициентами, зависящими от температуры:

$$
\begin{gathered}
c \rho \frac{\partial T}{\partial t}=\frac{\partial}{\partial x}\left(\lambda \frac{\partial}{\partial x} T\right), \\
T(x, 0)=T_{0} \\
-\left.\lambda \frac{\partial T}{\partial x}\right|_{x=0}=j \\
T(L, t)=T_{0} .
\end{gathered}
$$

Значение потока $j=P / S$, где $P-$ известная из эксперимента величина мощности нагревателя, $S$ известное значение площади нагревателя.

В задаче (1) использованы коэффициенты: $c$ - удельной теплоемкости, $\rho-$ плотности вещества, $\lambda-$ коэффициента теплопроводности.

Для решения данной системы уравнений использовался неявный численный метод контрольных объемов на равномерной сетке $[14,15]$. Соответствующая численная схема для уравнения (1) в $n$-ом узле сетки записана в виде:

$$
\begin{gathered}
c_{i} \rho_{i} \frac{T_{i}^{n+1}-T_{i}^{n}}{\tau}=-\left[-T_{i+1}^{n+1} \frac{2 \lambda_{i} \lambda_{i+1}}{\left(\lambda_{i}+\lambda_{i+1}\right)}\right. \\
+T_{i}^{n+1}\left(\frac{2 \lambda_{i} \lambda_{i+1}}{\left(\lambda_{i}+\lambda_{i+1}\right)}+\frac{2 \lambda_{i} \lambda_{i-1}}{\left(\lambda_{i}+\lambda_{i-1}\right)}\right) \\
\left.-T_{i-1}^{n+1} \frac{2 \lambda_{i} \lambda_{i-1}}{\left(\lambda_{i}+\lambda_{i-1}\right)}\right] / \Delta x^{2} .
\end{gathered}
$$

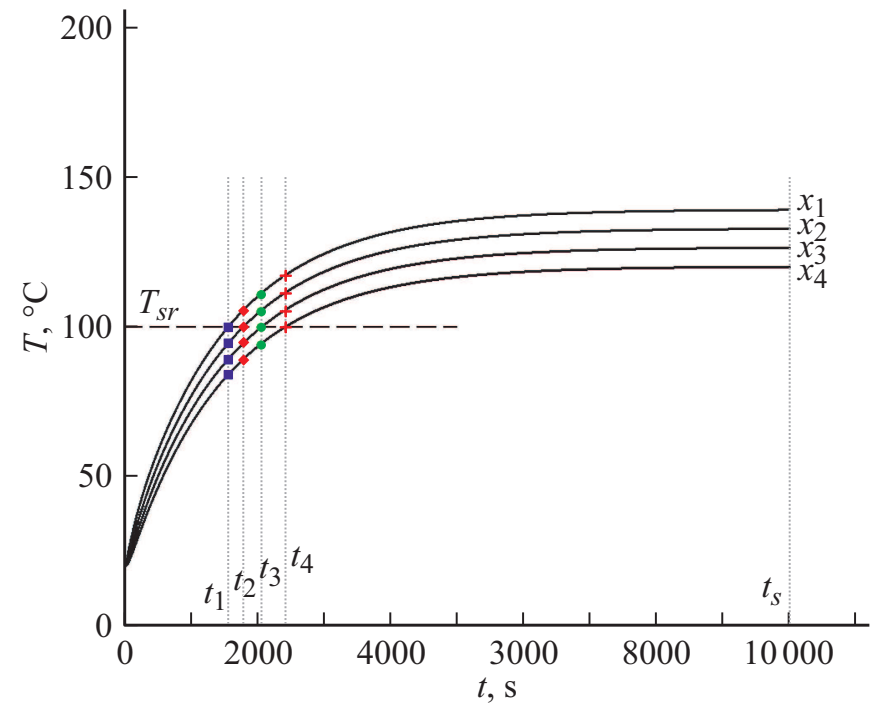

Рис. 1. Зависимость показаний термопар $T$ от времени $t$; $t_{s}$ - момент времени выхода на квазистационарный режим, $T_{s r}$ - температура среза кривых значений термопар в моменты времени, $t_{1}, t_{2}, t_{3}, t_{4}, x_{1}, x_{2}, x_{3}, x_{4}-$ местоположение термопар.

Здесь $\tau$ - шаг по времени, $\Delta x-$ шаг по сетке, $i-$ номер узла на сетке, характеризующий местоположение координаты $x_{i}, T_{i}$ - значение температуры в узле $i$, $n$ - временной слой, $c_{i}$ - значение теплоемкости, $\rho_{i}-$ плотности, $\lambda_{i}-$ коэффициента теплопроводности при температуре $T_{i}^{n}$. Численная схема (2) позволяет итерационно найти решение краевой задачи (1) в виде набора значений $T\left(x_{i}, t\right)$, при этом значения коэффициентов $c(T), \rho(T), \lambda(T)$ на каждой итерации определяются по распределениям температуры, полученным в предыдущих итерациях.

В численном эксперименте выберем четыре пространственных точки вдоль длины стержня, в которых происходит запись значений температуры в произвольные моменты времени с помощью виртуальных „термопар“. Размеры термопар предполагаются достаточно малыми и не влияющими на процесс распространения тепла. Предполагается, что в реальных (натурных) экспериментах в этих точках будут располагаться измерительные термопары. Местоположение термопар отметим координатами $x_{1}, x_{2}, x_{3}, x_{4}$. На рис. 1 для примера представлены рассчитанные зависимости показаний термопар (т.е. температур в выбранных точках) от времени. Качественно все такие зависимости представляют собой кривые насыщения, стремящиеся к стационарному значению с течением времени.

\section{3. Методика определения коэффициента теплопроводности для стационарного режима}

После проведения имитационного моделирования имеется набор термических кривых, которые были по- 
лучены в некоторых точках пространства (термопарах). На данном этапе уже не предполагается знания теплофизических параметров исследуемого материала: значения коэффициентов теплопроводности и температуропроводности, использованные в имитационном моделировании, в дальнейшем будут использованы лишь для оценки точности предлагаемого метода.

Учитывая результаты имитационного моделирования, постановка задачи выглядит следующим образом. Имеем набор термических кривых в точках пространства $x_{1}$, $x_{2}, x_{3}, x_{4}$. Необходимо определить теплофизические параметры (предполагаемого неизвестным) исследуемого материала.

Рассмотрим момент времени, когда система достаточно близка к равновесию. Интегрирование уравнения (1) по пространству в стационарном режиме приводит к уравнению

$$
-\lambda \frac{\partial T}{\partial x}=j,
$$

которое справедливо в любой точке пространства одномерного стержня.

При заданной величине теплового потока $j$ в уравнении (3) распределение температуры в стержне в этот момент времени $t_{s}$ (квазистационарный режим) слабо меняется со временем, т.е. $\Delta T\left(x_{k}, t_{s}\right) /\left(T\left(x_{k}, t_{s}\right) \ll 1\right.$, и поток $j$ почти постоянен вдоль стержня. Зафиксируем в момент времени $t_{s}$ одновременные значения температур $T_{k}$ в точках $x_{k}$, где $k-$ номер термопары, $x_{k}-$ местоположение термопары (рис. 1).

Чтобы выбрать наиболее удачную рациональную аппроксимацию для функции ехр на отрезке, аппроксимируем температуру по координате (вдоль стержня) в момент времени $t_{s}$ дробно-рациональной функцией Паде [16]:

$$
f(x)=\frac{a_{0}+a_{1} x+\ldots+a_{L} x^{L}}{b_{0}+b_{1} x+\ldots+b_{M} x^{M}} .
$$

Выберем $b_{0}=1$, чтобы убрать произвол в выборе параметров, и ограничимся в функции (4) коэффициентами Паде $a_{0}, a_{1}, b_{1}, b_{2}$. Восстановим их по показаниям термопар $\left(x_{k}, T_{k}\right)$ в четырех точках пространства, и запишем аппроксимирующую функцию для температуры и пространственной производной

$$
\begin{gathered}
T(x)=\frac{a_{0}+a_{1} x}{1+b_{1} x+b_{2} x^{2}}, \\
\frac{\partial T}{\partial x}=\frac{a_{1}}{1+b_{1} x+b_{2} x^{2}}-\frac{\left(a_{0}+a_{1} x\right)\left(b_{1}+2 b_{2} x\right)}{\left(1+b_{1} x+b_{2} x^{2}\right)^{2}} .
\end{gathered}
$$

Используя данные численного эксперимента, $T_{k}$, а так же значения координат термопар, найдем коэффициенты уравнения

$$
T_{k}=\frac{a_{0}+a_{1} x_{k}}{1+b_{1} x_{k}+b_{2} x_{k}^{2}} .
$$

Решение системы из четырех уравнений (7) относительно четырех коэффициентов $a_{0}, a_{1}, b_{1}, b_{2}$ позволяет подставить их значения в уравнение (6), аналитически определяющее производную температуры в каждой точке пространства. Тогда коэффициент теплопроводности определяется из закона Фурье как

$$
\lambda=-j /\left(\frac{\partial T}{\partial x}\right)
$$

\section{4. Методика определения коэффициента температуропроводности в нестационарном режиме}

На рис. 1 линия $T=T_{s r}$ пересекает кривые значений термопар в некоторые моменты времени $t_{1}, t_{2}, t_{3}, t_{4}$. В эти моменты времени были вычислены значения функции распределения температуры в стержне $T\left(x, t_{1}\right)$, $T\left(x, t_{2}\right), T\left(x, t_{3}\right), T\left(x, t_{4}\right)$, используя аппроксимации Паде, аналогично тому, как это было сделано при определении коэффициента теплопроводности.

По данным зависимостям находятся первые и вторые производные по пространству

$$
\begin{gathered}
\left.\frac{\partial T\left(x, t_{k}\right)}{\partial x}\right|_{x=x_{k}, T=T_{s r}}, \\
\left.\frac{\partial^{2} T\left(x, t_{k}\right)}{\partial x^{2}}\right|_{x=x_{k}, T=T_{s r}},
\end{gathered}
$$

где $k=1, \ldots 4$.

Из уравнения (1), используя данные с двух термопар, получаем систему из двух уравнений с двумя неизвест-

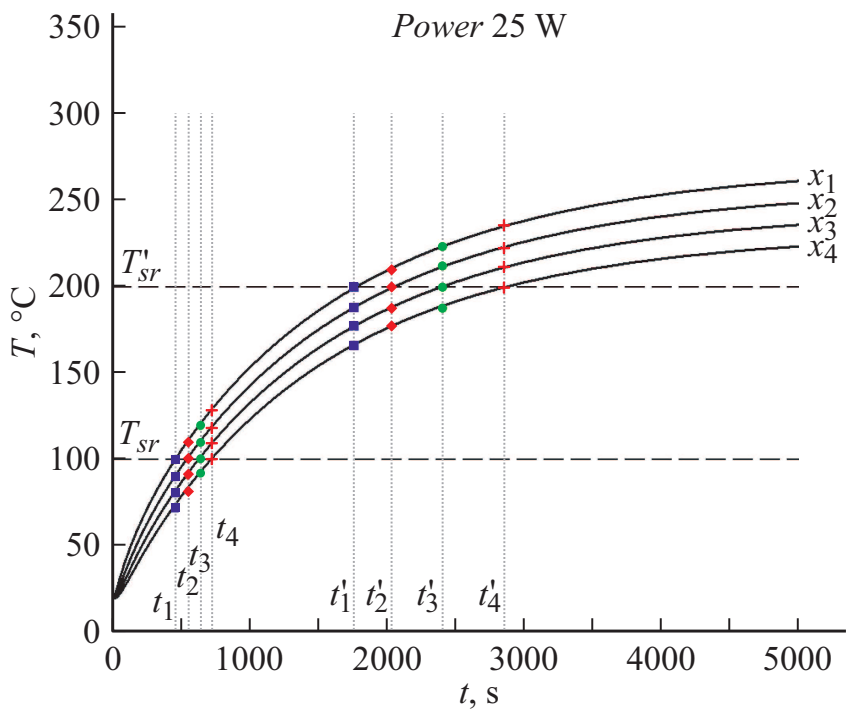

Рис. 2. Термические кривые $T(t)$ от времени $t$ при заданной мощности $P=25 \mathrm{~W}$ для метода прямого нагрева. 

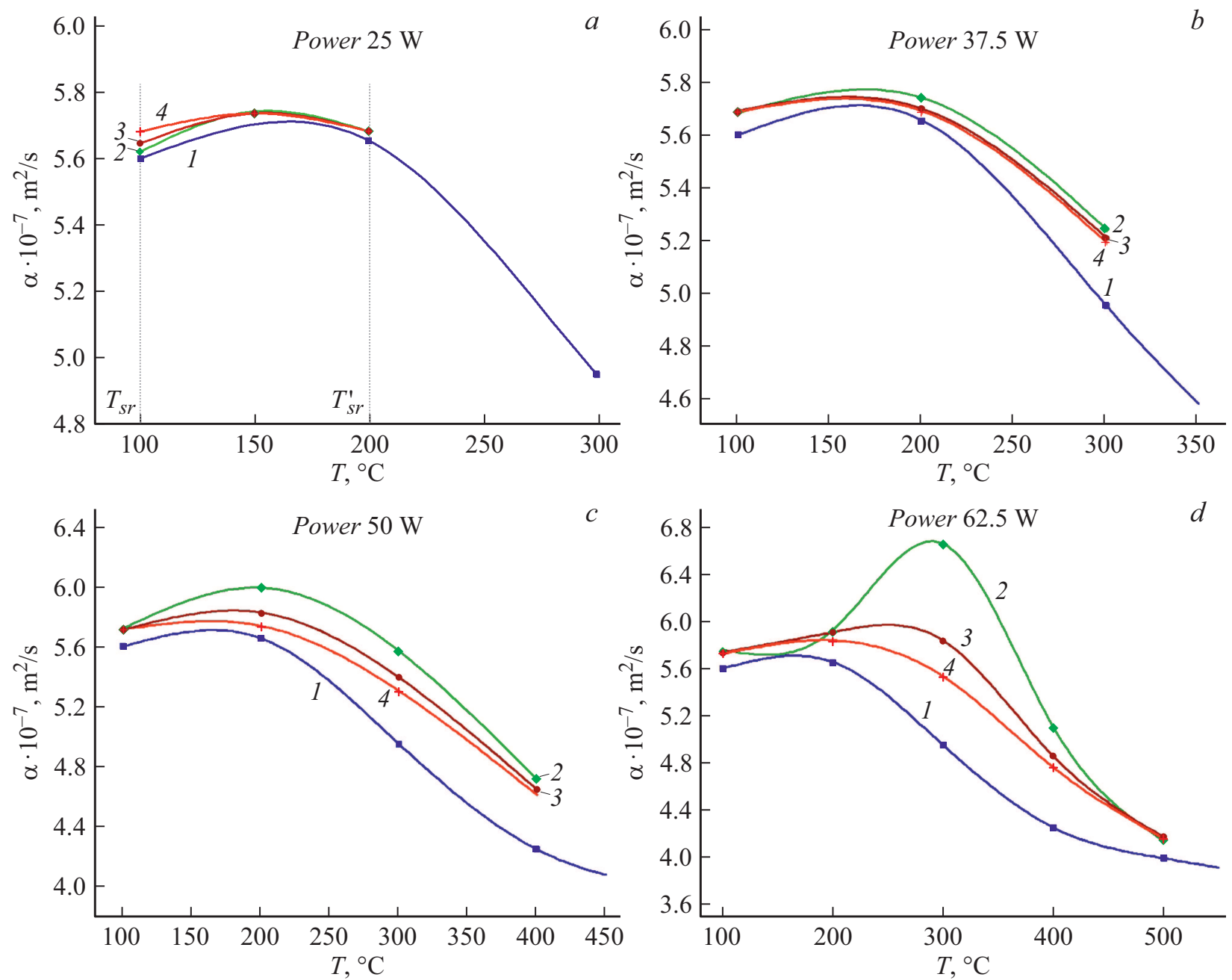

Рис. 3. Зависимости коэффициента температуропроводности $\alpha$ от температуры $T: 1$ - значение коэффициента температуропроводности от температуры, взятое из базы данных, 2 - значение коэффициента температуропроводности от температуры, рассчитанное по термическим кривым, расположенными на расстоянии 6.5 и $8.5 \mathrm{~mm}$ от источника, 3 - по термическим кривым, расположенными на расстоянии 8.5 и $10.5 \mathrm{~mm}$ от источника, 4 - по термическим кривым, расположенными на расстоянии 10.5 и $12.5 \mathrm{~mm}$ от источника. Мощность нагревателя задавалась от 25 до $62.5 \mathrm{~W}$.

ными $\alpha, \beta$ :

$$
\begin{gathered}
\frac{\partial T(x, t)}{\partial t}=\beta\left(\frac{\partial T(x, t)}{\partial x}\right)^{2}+\alpha \frac{\partial^{2} T(x, t)}{\partial x^{2}}, \\
\text { при } x=x_{1}, \quad t=t_{1}, \quad T(x, t)=T_{s r}, \\
\frac{\partial T(x, t)}{\partial t}=\beta\left(\frac{\partial T(x, t)}{\partial x}\right)^{2}+\alpha \frac{\partial^{2} T(x, t)}{\partial x^{2}}, \\
\text { при } x=x_{2}, \quad t=t_{2}, \quad T(x, t)=T_{s r},
\end{gathered}
$$

где $\alpha=\alpha / c \rho, \beta=\frac{1}{c \rho} \frac{\partial \lambda}{\partial T}$ определяются при температуpe $T_{s r}$.

Разрешая уравнение (10) относительно $\alpha, \beta$, вычисляем коэффициент температуропроводности $\alpha$. С учетом того что коэффициент теплопроводности $\lambda$ уже вычислен, последнее позволяет определить значения коэффициента теплоемкости при соответствующих температурах.

\section{5. Определение коэффициента теплопроводности в нестационарном режиме}

Большие затраты времени на определение коэффициента теплопроводности в стационарном режиме заставляют задуматься о том, как измерять коэффициент теплопроводности в нестационарном случае. Выход может состоять в использовании системы уравнений

$$
\begin{gathered}
\alpha=\frac{\lambda}{c \rho}, \\
\beta=\frac{1}{c \rho} \frac{\partial \lambda}{\partial T},
\end{gathered}
$$

где $\alpha$ и $\beta-$ функции, зависящие от температуры $T$ и входящие в уравнение (10). 

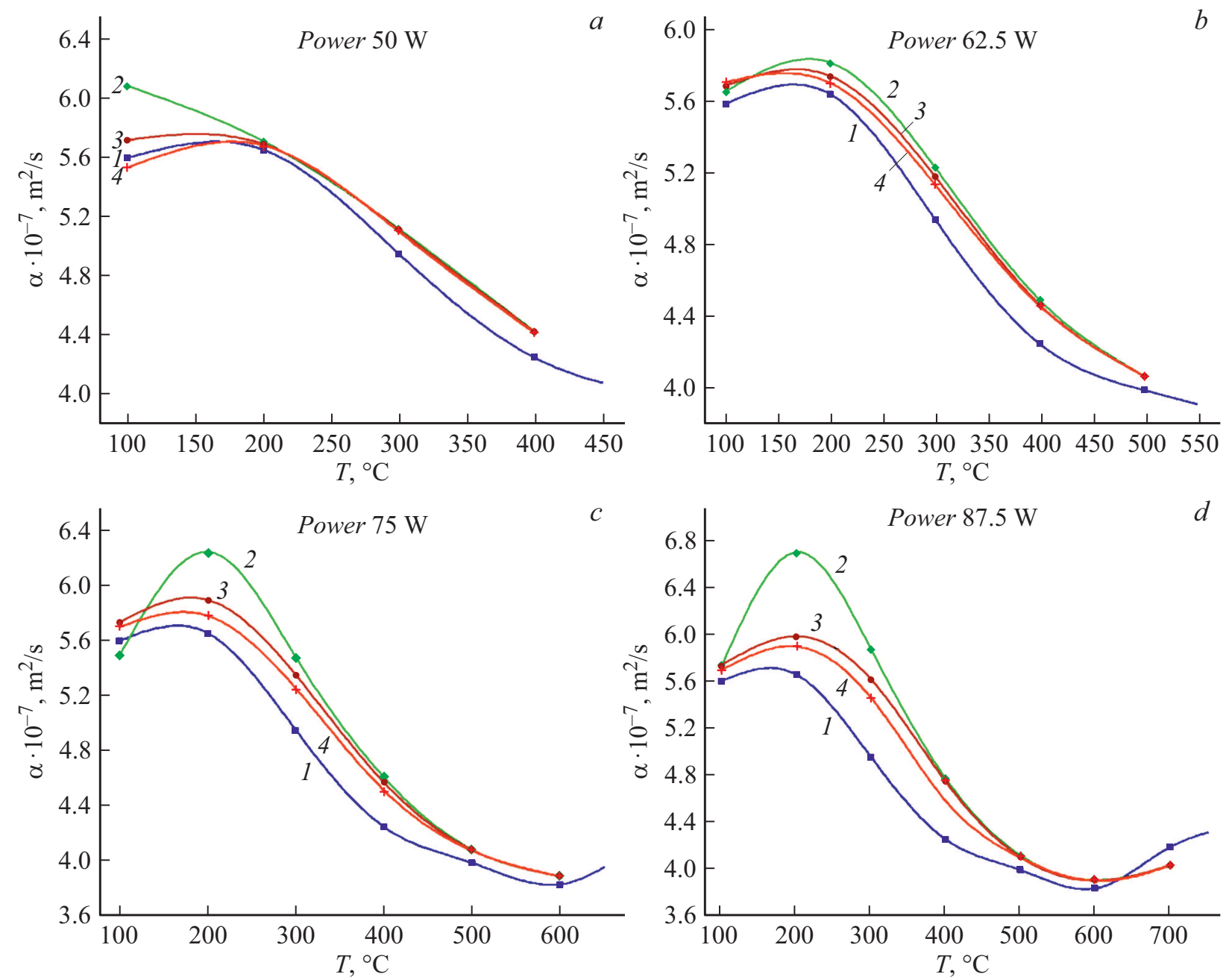

Рис. 4. Зависимость коэффициента температуропроводности $\alpha$ от температуры $T: 1$ - значение коэффициента температуропроводности от температуры, взятое из базы данных, 2 - значение коэффициента температуропроводности от температуры, рассчитанное по термическим кривым, расположенными на расстоянии 10.5 и $11.5 \mathrm{~mm}$ от источника, 3 - по термическим кривым, расположенными на расстоянии 11.5 и $12.5 \mathrm{~mm}$ от нагревателя, 4 - по термическим кривым, расположенными на расстоянии 12.5 и $13.5 \mathrm{~mm}$ от нагревателя Расстояние между термопарами $1 \mathrm{~mm}$, мощность нагревателя от 50 до $87.5 \mathrm{~W}$.

Из соотношений (11) легко получить дифференциальное уравнение, решение которого будет иметь вид

$$
\lambda=\lambda_{0} \exp \left(\int_{T_{0}}^{T} \frac{\beta(T)}{\alpha(T)} d T\right),
$$

где $\lambda_{0}-$ значение коэффициента теплопроводности при $T_{0}$. Вычисление коэффициента $\lambda_{0}$ в настоящей работе представлено в методике определения коэффициента теплопроводности для стационарного режима. После однократного стационарного измерения $\lambda_{0}$ далее при интегрировании по температуре значения $\lambda$ находятся по известным зависимостям $\alpha$ и $\beta$.

Такой подход мог бы существенно ускорить процесс измерений теплофизических параметров, но необходимо учесть, что при непосредственном интегрировании соотношения (12) по полученным данным возможно значительное накопления ошибок. Поэтому в настоящей работе мы избегаем использования соотношения (12), предполагая к нему вернуться в следующей работе.

\section{6. Постановка численных экспериментов}

На основе термических кривых имитационного моделирования (рис. $2,5,7)$ были вычислены теплофизические свойства (рис. $3,4,8$ ). Численные эксперименты выполнены в двух постановках.

1. „Прямой метод“. На рис. 2 представлен результат численного эксперимента, который получен ,прямым“ методом. Он заключается в том, что задается определенная мощность нагревателя-источника, в данном случае $P=25 \mathrm{~W}$. В исследуемом материале устанавливаются термопары на выбранном расстоянии от источника. Ре- 


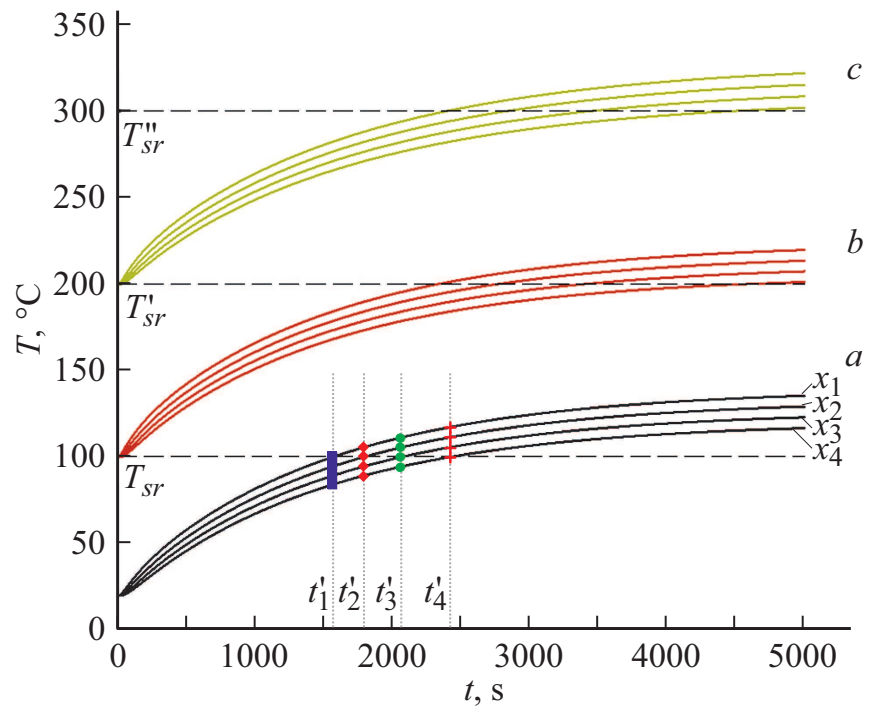

Рис. 5. Термические кривые $T(t)$ для метода прогрева формы при заданной мощности $P=12.5 \mathrm{~W}$ для всех численных экспериментов $-a-c$ и т.д. Всего экспериментов 15 .

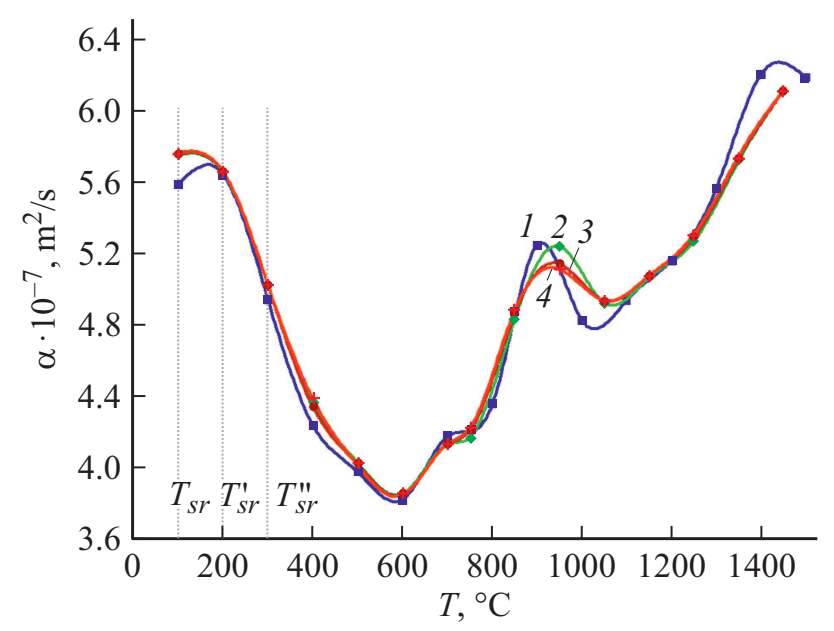

Рис. 6. Зависимость коэффициента температуропроводности $\alpha$ от температуры $T$, полученная методом прогрева формы, при мощности $P=12.5 \mathrm{~W}: 1$ - значение температуропроводности от температуры, взятое из базы данных, 2 - значение температуропроводности от температуры, рассчитанное по термическим кривым, расположенными на расстоянии 6.5 и $8.5 \mathrm{~mm}$ от источника нагрева, 3 - по термическим кривым, расположенными на расстоянии 8.5 и $10.5 \mathrm{~mm}$ от источника нагрева 4 - по термическим кривым, расположенными на расстоянии 10.5 и $12.5 \mathrm{~mm}$ от источника нагрева

шая уравнение (2), получаем результат в виде графиков зависимости температур от времени $T(t)$. Используя представленную выше методику определения коэффициента температуропроводности и обрабатывая результаты численного эксперимента, получаем зависимости коэффициента температуропроводности от температуры. Результаты изображены на рис. 3, 4 .
2. „Метод прогрева формы“. Результат численного эксперимента представлен на рис. 5. Мощность источника осталась постоянной и равной $P=12.5 \mathrm{~W}$. Менялись граничные условия, начальная температура и температура исследуемого материала на $100^{\circ} \mathrm{C}$ в каждом следующем численном эксперименте $-a-c$ и т.д.

Всего было проведено 15 таких измерений. Используя методику определения коэффициента температуропроводности, все вычисленные значения коэффициента температуропроводности от температуры каждого численного эксперимента заносились в одну таблицу. Затем строился график зависимости коэффициента температуропроводности от температуры (рис. 6).

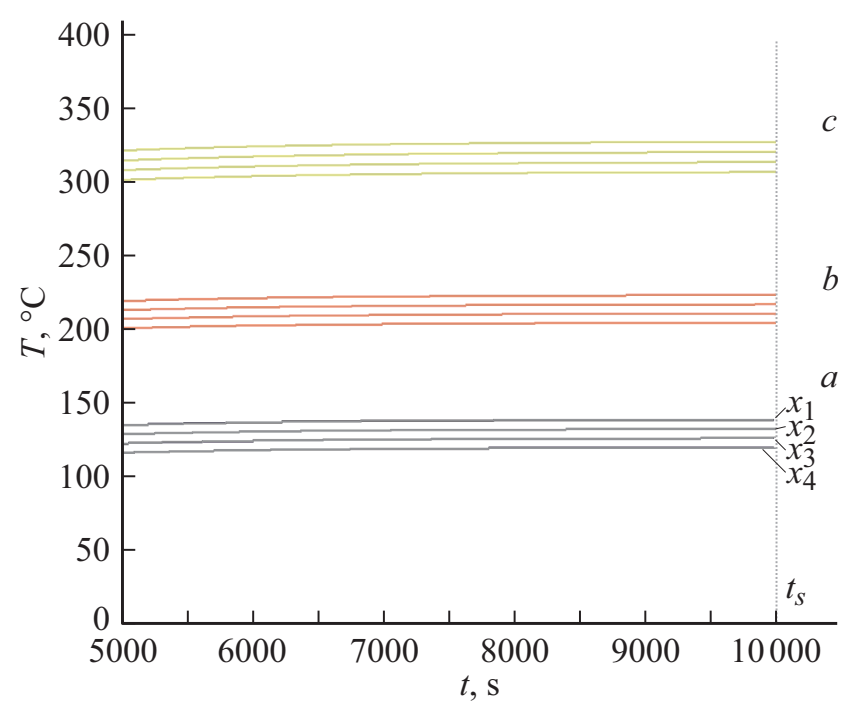

Рис. 7. Термические кривые $T(t)$ для метода прогрева формы в области квазистационара при заданной мощности $P=12.5 \mathrm{~W}$ для всех численных экспериментов $a-c$.

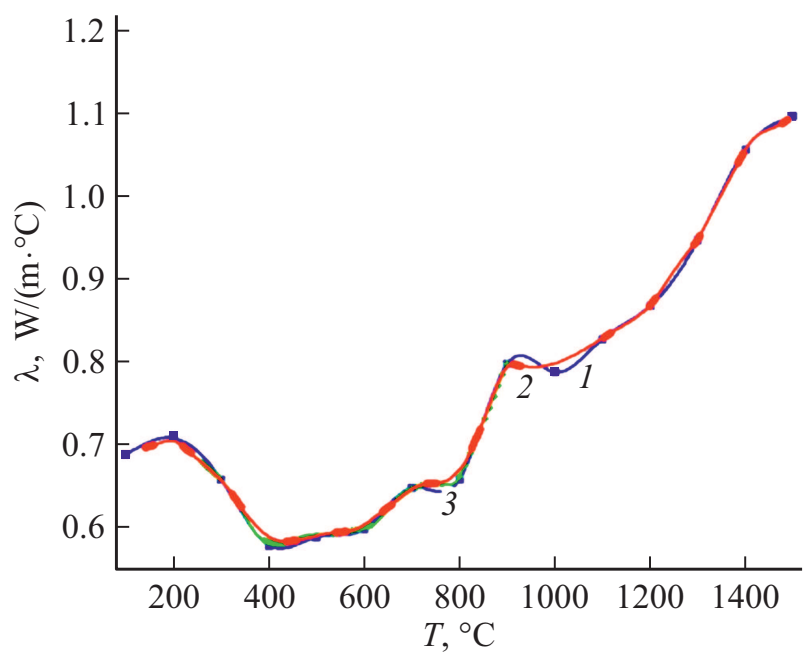

Рис. 8. Зависимости коэффициентов теплопроводности $\lambda$ от температуры $T: 1-$ значения, взятые из базы данных, $2-$ значения, полученные из метода прогрева, 3 - значения, полученные из прямого метода. 


\section{7. Результаты}

При анализе результатов численных экспериментов прямого нагрева было выявлено следующее:

1. Чем выше мощность нагревателя, тем больше разница значений температур в соседних термопарах в один момент времени, тем самым растет ошибка в определении коэффициента температуропроводности.

2. В исследуемом материале, чем дальше от источника расположены термопары, тем равномернее показания температурных данных, следовательно, при определении коэффициента температуропроводности ошибка расчетов, снимаемых с дальних термопар, ниже. При этом уменьшение расстояния между термопарами тоже вносит вклад в уменьшении ошибки в определении коэффициента температуропроводности.

3. При первом эксперименте (рис. 3) приемлемые результаты с максимальной ошибкой в 5\% были получены при подаваемой мощности $37.5 \mathrm{~W}$ и прогревом тестируемого материала до $300^{\circ} \mathrm{C}$.

4. Во втором эксперименте (рис. 4) удалось получить результаты с ошибкой чуть менее 6\% (5.8\%) при мощности в $75 \mathrm{~W}$ и прогревом материала до $600^{\circ} \mathrm{C}$, но только по значениям самых удаленных от нагревателя термопар.

Существует корреляция ошибки определения коэффициента температуропроводности с местоположением термопар и подаваемой мощностью нагревателя.

Методом прогрева формы исследуемый материал удалось прогреть до $1500^{\circ} \mathrm{C}$. Как видно из рис. 6, почти на всем температурном интервале получены значения с ошибкой не более $4 \%$, кроме смещения пика в районе $900^{\circ} \mathrm{C}$. Предполагается, что это смещение связано с резким изменением теплофизических свойств, и необходимо делать расчет с меньшим шагом по температуре прогрева материала.

Коэффициент теплопроводности вычислялся по методике определения этой величины в стационарном случае. Для этого брались термические кривые в области квазистационара (насыщения). Например, на рис. 7 представлены термические кривые, значения температур которых стремятся к стационарному поведению (на последнем расчетном шаге по времени изменение значений температур происходит в 4 знаке после запятой), полученные методом прогрева формы.

На рис. 8 представлены зависимости значений коэффициента теплопроводности от температуры.

Значения коэффициента теплопроводности, вычисленные при разных численных экспериментах, близки к исходным значениям, заложенным в имитационную модель, так что относительное отклонение на всем интервале температур не превышает 3\%.

\section{Выводы}

В результате работы рассмотрены две методики определения коэффициента температуропроводности исследуемого материала: метод „прямого нагрева“ и метод „прогрева формы“. Расчет теплопроводности был выполнен в стационарном режиме. Необходимые экспериментальные данные были получены из имитационного моделирования исследуемого материала с помощью численного решения одномерной задачи уравнения теплопроводности. Значения коэффициентов теплопроводности и температуропроводности рассчитаны по данным значениям термических кривых, полученных из имитационного моделирования. Поскольку значения коэффициентов теплопроводности и температуропроводности рассчитаны независимо друг от друга, наличие двух независимых величин позволяет определить значение удельной теплоемкости материала.

Значения коэффициента теплопроводности, вычисленные при различных численных экспериментах, близки к исходным данным имитационного моделирования: относительное отклонение на всем интервале температур не превышает 3\%.

Используя метод „прямого нагрева“ с небольшими мощностями нагревателя и подобрав наилучшее местоположение термопар, можно получить приемлемые результаты до температуры порядка $600^{\circ} \mathrm{C}$. При увеличении мощности нагревателя используемый метод требует дополнительных исследований. Это связано как с сильной нелинейностью физических процессов, так и с неуниверсальностью математического аппарата, который начинает вносит существенный вклад в общую ошибку при обработке численных результатов эксперимента.

Подход, связанный с предварительным прогревом формы в каждом расчете и постоянной мощностью, позволяет определять коэффициент температуропроводности с относительным отклонением не более $4 \%$, даже при высоких температурах.

Результат исследования дает качественные представления о том, как меняются теплофизические свойства материалов при различных режимах, а также позволяет оценить погрешность полученных параметров за счет сравнения с данными, заложенными в имитационное моделирование.

В дальнейшем планируется исследовать влияние выбора методов аппроксимаций для задачи определения теплофизических параметров материалов.

\section{Финансирование работы}

Исследование выполнено при частичной финансовой поддержке РФФИ и Правительства Удмуртской Республики в рамках научного проекта № 18-42-180002. 


\section{Конфликт интересов}

Авторы заявляют, что у них нет конфликта интересов.

\section{Список литературы}

[1] N. Yüksel. The Review of Some Commonly Used Methods and Techniques to Measure the Thermal Conductivity of Insulation Materials. DOI: https://doi.org/10.5772/64157

[2] А.А. Самарский, П.Н. Вабищевич. Численные методы решения обратных задач математической физики. (ЛКИ, М., 2009). В.С. Зарубин. Математическое моделирование в технике. (МГТУ им. Н.Э. Баумана, М., 2001).

[3] О.М. Алифанов. Идентибикация процессов теплообмена летательных аппаратов (введение в теорию обратных задач теплообмена). (Машиностроение, М., 1979).

[4] В.Ф. Формалев, С.А. Колесник. ТВТ, 55 (4), 564 (2017). DOI: https://doi.org/10.7868/S0040364417040068

[5] В.Ф. Формалев, С.А. Колесник. ТВТ, 51 (6), 875 (2013). DOI: https://doi.org/10.7868/S0040364413050062

[6] V.F. Formalev, S.A. Kolesnik. Intern. J. Heat Mass Transfer, 123, 994 (2018). DOI: https://doi.org/10.1016/j.ijheatmasstransfer.2018.03.014

[7] N. Yüksel, A. Avci, M. Kiliç. Heat Mass Transfer, 48, 1569 (2012). DOI: https://doi.org/10.1007/s00231-012-1001-2

[8] Saylor Academy. Thermal conductivity. 2016. Available from: http://www.saylor.org/site/wp.content/uploads/2011/04/Thermal_conductivity.pdf[Accessed: 2019.12.8]

[9] K123 of the department of materials engineering and chemistry. Chapter 16. Determination of Thermal Conductivity [Internet]. 2016. Available from: http://tpm.fsv.cvut.cz/ student/documents/files/BUM1/Chapter16.pdf[Accessed: 2019.12.8]

[10] N.N. Mohesnin editor. Thermal Properties of Food and Agricultural Materials, 1st ed. (Gordon and Breach Science Publishers, NY, 1980). DOI: $10.2307 / 2530323$

[11] H. Czichos, T. Saito, L.E. Smith editors. Springer Handbook of Materials Measurement Method, 1st ed. (Springer Science \& Business Media, NY., 2006). DOI: $10.1007 / 978.3 .540 .30300 .8$

[12] http://magma.maths.usyd.edu.au/magma/ (от 15.04.2020), https://www.esi-group.com/ (om 15.04.2020), http://lvmflow.ru/ \& https://www.novacast.se/ (om 15.04.2020)

[13] Д. Андерсон, Дж. Таннехил, Р. Плетчер. Вычислительная гидромеханика и теплообмен, в 2-х т. (Мир, М., 1990) T. 1.

[14] А.А. Самарский. Теория разностных схем. (Наука, М., 1982).

[15] Дж. Бейкер мл., П. Грейвс-Моррис. Аппроксимащии Паде. (Мир, М., 1986). 